\title{
LOS ESTEREOTIPOS ÉTNICOS EN LOS PROFESIONALES DEL DEPORTE
}

\author{
ETHNIC STEREOTYPES IN SPORTS AND PHYSICAL ACTIVITY \\ PROFESSIONALS
}

OS ESTEREÓTIPOS ÉTNICOS NOS PROFISSIONAIS DO ESPORTE

Mascia Migliorati*, Antonio Fraile Aranda**, Rufino Cano González ${ }^{* *}$

Palabras clave

Enseñanza.

Deportes. Atletas.

Grupo con

ascendencia del

continente africano.
Resumen: El objetivo del artículo es conocer si los actuales y futuros profesionales del deporte poseen estereotipos étnicos respecto a los deportistas negros. Entrevistamos a 38 entrenadores profesionales y estudiantes de Educación Física y aplicamos un cuestionario a 272 estudiantes de tres Facultades de Ciencias Motoras de la región italiana del Lacio. Los resultados muestran que, entre los entrenadores y estudiantes de Educación Física, existen estereotipos étnicos, ligados a la pertenencia étnica y en relación con el rendimiento deportivo, tales como la creencia de superioridad y la predisposición biológico-genética de los deportistas negros para el baloncesto o para la carrera de velocidad, así como una menor predisposición para la natación. Esto supone elaborar una propuesta formativo-pedagógica sobre las implicaciones educativas de ciertos estereotipos entre los escolares.

Abstract: The main aim of this paper is to know whether current and future sports professionals hold ethnic stereotypes about black athletes. We interviewed a group of 38 professional coaches and Physical Education students, and administered a questionnaire to a sample of 272 students from three Movement and Health Science departments in the Italian region of Lazio. The results show that both coaches and sport science students hold ethnic stereotypes on ethnic belonging and connected to concepts of sports performance, such as the belief in biological superiority and genetic predisposition of black athletes for basketball or sprinting and their lower inclination to swimming. The study highlights the need for a pedagogical proposal on the educational implications of certain stereotypes for young students.

Resumo: 0 objetivo do artigo é saber se os atuais e futuros profissionais do esporte têm estereótipos étnicos a respeito dos desportistas negros. Entrevistamos 38 treinadores profissionais e estudantes de Educação Física e aplicamos um questionário a 272 estudantes de três Faculdades de Ciências Motoras da região italiana de Lácio. Os resultados mostram que, dentre os treinadores e estudantes de Educação Física, existem estereótipos étnicos ligados à procedência étnica e em relação ao rendimento desportivo, tais como a crença de superioridade e a predisposição biológico-genética dos desportistas negros para o basquete e para a corrida de velocidade, assim como uma menor predisposição para a natação. Isto supõe elaborar uma proposta formativopedagógica sobre as implicações educativas de certos estereótipos dentre os escolares.
* Universitad de Roma Foro Itálico, Roma, Itália.

E-mail: mmigliorati@sorrisinelmondo.it

** Universidad de Valladolid. Valladolid, Espanha.

E-mail: afraile@mpc.uva.es; rcano@pdg.uva.es

Recebido em: 20-10-2015 Aprovado em: 13-02-2015 


\section{INTRODUCCIÓN}

En este estudio existen dos conceptos clave que conviene aclarar y distinguir: raza y etnia. El concepto de "raza" afecta a una categoría de individuos que comparten ciertos rasgos hereditarios socialmente significativos (MACIONIS, 2007). En biología, este término ha sido usado para definir a grupos de individuos diferentes dentro de una misma especie. Se refiere a una cualidad constante y transmisible, determinada genéticamente, que está progresivamente desapareciendo de la terminología científica de la antropología, de la biológica y de la genética humana (BARBUJANI, 2006).

El concepto "etnia" se refiere al grupo de personas que comparten una misma herencia cultural, tienen antepasados comunes, hablan una misma lengua, practican la misma religión, comparten costumbres e identidad social. Mientras que el primero incide más en factores biológicos, el segundo posee un carácter cultural.

En nuestro caso, para distinguir las personas de los diferentes grupos étnicos objeto de estudio, utilizaremos el término "blanco" para aquellas personas de Europa y del Medio Oriente y, el término "negro o afro-americano", para los grupos raciales negros de África. Ambas categorías son construcciones sociopolíticas y no deben interpretarse como científicas 0 antropológicas.

Los estereotipos se han ido construyendo a partir de teorías que explican distintas prestaciones deportivas entre afro-americanos y americanos de origen europeo (WIGGINS, 1989; HOBERMAN, 1997). Aunque algunos estudios han encontrado diferencias anatómicas y fisiológicas entre blancos y negros, estos se basan en muestras limitadas y no vinculadas al rendimiento deportivo (HUNTER, 2009). Más allá de un debate científico, entre los investigadores que afirman la realidad biológica sustancial del concepto de "raza" y los que subrayan que "la raza" es un puro constructo social, es importante destacar que la práctica deportiva suele estar más vinculada a factores culturales y no tanto a diferencias biológicas. Estas diferencias son mínimas y no justifican su rendimiento deportivo (HARRISON, 2001), vinculándose, más bien, a una serie de factores relacionados con la construcción de la identidad personal, la educación y el entorno social. Por tanto, las categorías para clasificar, a nivel racial, los grupos humanos son imprecisas y completamente arbitrarias (BARBUJANI, 2006).

En el escenario deportivo, algunos jóvenes deportistas y sus entrenadores creen en la superioridad de algunos grupos étnicos sobre otros (HARRISON, 2001). Igualmente, existe el tópico de que las personas negras nadan peor debido a que tienen huesos más pesados que los blancos, aunque están más predispuestos para los deportes de fuerza física y explosividad. Estos juicios representan estereotipos étnicos, es decir, creencias incompletas y generalizadas hacia un grupo social determinado (ALLPORT, 1954) sin fundamento científico comprobado, ya que son construcciones sociales. Esos juicios son difícilmente modificables y tienden a autorreproducirse (MAZZARA, 1997).

Investigaciones realizadas por (SAILES, 1993; HAYES; SUDGEN, 1999; JOHNSON, HALLINAN; WESTERFIELD, 1999; BURDEN, HODGE; HARRISON, 2004; HARRISON; LAWRENCE, 2004; SHELDON, JAYARATNE; PETTY, 2007; AZZARITO; HARRISON, 2008; HODGE et al., 2008; HARRISON; LAWRENCE; BUKSTEIN, 2011) han evidenciado la presencia de estereotipos vinculados al determinismo biológico (los negros están dotados de manera natural para el deporte), o que los negros presentan ventajas naturales para algunos deportes 
(baloncesto, carrera de velocidad y boxeo), y desventajas para otros (natación, tenis y golf). Igualmente, SAILES (1993); HARRISON, AZZARITO; BURDEN (2004); HODGE et al. (2008) y HARRISON et al., (2011) señalan que los atletas de raza negra son mejores.

En un estudio de Hayes y Sudgen (1999), con 39 profesores ingleses de Educación Física, con experiencia de al menos 5 años con alumnos negros en un contexto multiétnico, el $52 \%$ consideraba que los alumnos negros tenían un éxito deportivo desproporcionado, mientras que el $31 \%$ no compartía esta afirmación. Un $82 \%$ de estos profesores creía que los alumnos negros presentaban ventajas en algunos deportes (atletismo, baloncesto, fútbol, cricket y boxeo), frente al resto que no estaba de acuerdo. Por último, para el $74 \%$ de los entrenadores el éxito de los deportistas negros se debía a razones fisiológicas ( un mayor porcentaje de fibras que favorecía la velocidad).

Un estudio sobre los estereotipos étnicos con 248 estudiantes, de cuatro grupos étnicos diferentes, fue realizado por Johnson, Hallinan y Westerfield (1999) analizando cuáles eran los motivos de su éxito en el baloncesto universitario. Los deportistas negros puntuaron más alto en factores genéticos innatos (capacidad natural de salto, mayor longitud de brazos y piernas, presencia de un tejido muscular extra, velocidad y capacidad de reacción natural) que en los socioeconómicos. Por el contrario, los blancos registraron una puntuación más alta en factores socioeconómicos (inteligencia y capacidad de liderazgo, acceso a mejores entrenadores y técnicas, acceso a mejores instalaciones deportivas, esfuerzo y dedicación).

En el atletismo, Rasmussen, Turner e Esgate (2005) confirman la existencia de creencias estereotipadas en la carrera de velocidad. Con 36 estudiantes del Grado de Entrenador en la Universidad de Luton analizaron los motivos del éxito de los atletas blancos y negros en la carrera de velocidad. Los factores de éxito para los atletas blancos fueron: conocimiento y uso inteligente de los métodos de entrenamiento, acceso a mejores instalaciones, entrenamiento duro y acceso a mejores entrenadores. Para los atletas negros fueron: relajación y economía del movimiento, velocidad natural y capacidad de reacción, extremidades más largas y mayor masa muscular. Entre los atletas negros destacaban más los factores genéticos innatos que los socioeconómicos; a la inversa que en los atletas blancos. Finalmente, entre los futuros entrenadores existe la creencia de que los negros poseen habilidades atléticas innatas para la velocidad.

Turner e Jones (2007) recogen de un grupo de entrenadores de atletas velocistas que, para ellos, los atletas negros destacan por: una mayor predisposición fisiológica para la carrera, un mayor porcentaje de fibras para la velocidad y un fuerte vínculo entre los afrocaribeños y la carrera de velocidad. Esos estereotipos étnicos vinculados a los deportistas negros están presentes en los estudiantes, entrenadores y profesores de Educación Física, pudiendo altamente condicionar, no sólo el desarrollo del joven como deportista sino, también, como ciudadano. ${ }^{1}$

Considerando que en este artículo se trata de analizar las influencias negativas de la presencia de estereotipos, no tanto a nivel de alto rendimiento deportivo, como desde el deporte como herramienta facilitadora de interrelación e inclusión social. Se debe resaltar que los estereotipos priman en el ámbito de las actividades físicas afectando, negativamente, en la 
iniciación de los jóvenes deportistas y en la justa e igualitaria participación de las diferentes etnias en las múltiples disciplinas deportivas (HARRISON, 2001). Asimismo, las ideas estereotipadas influyen en el crecimiento, en el desarrollo y en la identificación de los adolescentes, representando un papel fundamental en la construcción de su identidad (HARRISON, 2001). Por tanto, guían las expectativas de los educadores deportivos condicionando, notablemente, las experiencias de aprendizaje (HORN; LOX, 1993), incluso influyendo en la calidad de las prestaciones deportivas (STONE et al. 1999). Así el deporte, que debería favorecer el encuentro intercultural (LLEIXÁ, 2004), puede incrementar la discriminación y el racismo (REFRIGERI, 2011; MIGLIORATI, 2012).

Para finalizar, esto nos obliga a preguntarnos: ¿Qué piensan y creen los profesionales del deporte, en el contexto italiano, sobre la etnicidad y el rendimiento deportivo? A partir de este interrogante, nuestra investigación pretende: "Descubrir si los actuales y futuros profesionales, en dicho contexto deportivo, poseen estereotipos étnicos relativos a los deportistas negros de baloncesto, atletismo (carrera de velocidad) y natación".

\section{METODOLOGÍA}

En una primera fase del estudio se entrevistaron a 38 entrenadores de las tres disciplinas deportivas referidas (19 entrenadores profesionales y 19 estudiantes/entrenadores de la Facultad de Ciencias Motoras de la Universidad "Foro Italico" de Roma) al objeto de identificar la posible presencia de estereotipos étnicos ligados a los deportistas negros en la carrera de velocidad, baloncesto y natación. Para atender al grado de credibilidad del estudio, la información obtenida y las categorías de análisis han sido sometidas al juicio de expertos, a la comprobación con los participantes y, finalmente, a una triangulación de estos indicadores de fiabilidad cualitativa, contrastando los datos y resultados obtenidos en otras investigaciones con estudios similares.

El conjunto de las categorías generales se concretan en dos: "naturaleza" y "cultura". Por su parte, el éxito atlético de los deportistas se puede explicar, por un lado, a partir del éxito deportivo basado en el talento natural-biológico del deportista y, por otro, a través del éxito deportivo dependiente, en gran parte, de las condiciones ambientales, económicas, sociales y motivacionales que rodean a los atletas. Ambos posicionamientos están, a menudo, ligadas a la raza.

Dentro de la categoría general "naturaleza", se estructuran tres subcategorías: a) ventaja física "raza" negra: indicando que los atletas negros son física y atléticamente superiores o poseen ventajas de carácter estructural, anatómico y muscular que, presumiblemente, les otorgan superioridad respecto a los deportistas blancos; b) desventaja física "raza" negra: sobre las presuntas características morfológicas, antropométricas, fisiológicas y motoras de los deportistas negros que, según los entrevistados, les producen desventajas en la práctica; c) inexistencia de ventaja/desventaja por "raza": niega la existencia de una ventaja 0 desventaja física debido a razones de pertenencia a un grupo étnico. Por ello, el éxito deportivo de un atleta está ligado a su talento individual más que al hecho de pertenecer a un grupo étnico determinado.

Dentro de la categoría general "cultura", y al objeto de identificar los factores que pueden condicionar el éxito o el fracaso deportivo, se establen las siguientes subcategorías:

a) factores socioculturales: incluye aquellos factores vinculados a la cultura y sociedad a la 
que pertenecen, como es el caso de una mayor difusión, en la comunidad de origen africano, de algunas disciplinas deportivas respecto de otras, o la influencia de la familia, del grupo de iguales y de los modelos deportivos a los que están expuestos los jóvenes desde pequeños; b) factores socioeconómicos: aquellos elementos vinculados con el estatus socioeconómico del atleta que pueden influir en la participación deportiva; c) factores motivacionales: opiniones de los sujetos que consideran que el deporte, para algunos deportistas negros, es el único medio de ascenso y de reconocimiento social.

Para la fase cuantitativa, se utiliza una escala de Likert, en una muestra de 272 estudiantes (34\% mujeres y $66 \%$ hombres), respetando la proporción por Universidad. El 22\% eran de la Facultad de Cassino, el $28 \%$ de la Facultad de Tor Vergata y el $50 \%$ de la Facultad Foro Itálico. Con una edad media de 22,2 años.

La validez de contenido del cuestionario se ha realizado mediante el juicio crítico de cuatro expertos y la aplicación de un "pretest" a un grupo de 30 estudiantes. En la validación de la fiabilidad, con un coeficiente alfa de Cronbach $(0,85)$, por tanto con un alto grado de precisión del instrumento. La información ha seguido un tratamiento descriptivo desde la aplicación estadística SPSS 20.0.

\section{RESULTADOS DE LA FASE CUALITATIVA}

a. Carrera de velocidad. Las unidades textuales muestran la opinión de los entrevistados sobre la superioridad atlética de los deportistas negros en esta disciplina, registrando una mayor proporción ( $n=56$ ) en la subcategoría "ventaja física raza negra". Algunos entrevistados han expresado sus opiniones vinculadas a aspectos de tipo biológico/genético:"Los atletas negros están, genéticamente, predispuestos a esta disciplina respecto a todas las demás etnias" (entrenador 25).

Son 30 los que consideran que el triunfo de los atletas negros en la carrera es debido a que poseen un sistema muscular más adaptado a esta disciplina: "Los atletas negros tienen un mayor número de fibras blancas con respecto a los blancos" (entrenadora 54).

Algunos menos perciben que el éxito deportivo en la carrera de velocidad se debe a características estructurales del cuerpo de los atletas negros: "El elemento estatura y la morfología física hacen que se tenga una predisposición natural a esta disciplina" (entrenador 37).

En el análisis de los resultados en la categoría "cultura" hay un total de 8 unidades textuales relacionadas con la subcategoría "factores socioculturales". Así, el éxito de los deportistas negros, en la carrera de velocidad, podría ser explicado por la mayor difusión de este deporte en la comunidad de origen africano: "El éxito de los atletas negros en esta disciplina es debido a la difusión de esta práctica en la comunidad de color" (entrenador 55).

Desde un análisis cualitativo, el $73,6 \%(n=28)$ considera que el motivo del éxito deportivo de los atletas negros en la carrera de velocidad se debe, exclusivamente, a factores de ventaja física, y, únicamente el 5,2\% $(n=2)$ argumenta aspectos de tipo sociocultural, de manera exclusiva. Por otro lado, el $15,7 \%(n=6)$ relaciona el éxito deportivo con factores de tipo genético y sociocultural (figura 1) 
Figura 1 - Motivos de éxito deportivo: carrera de velocidad

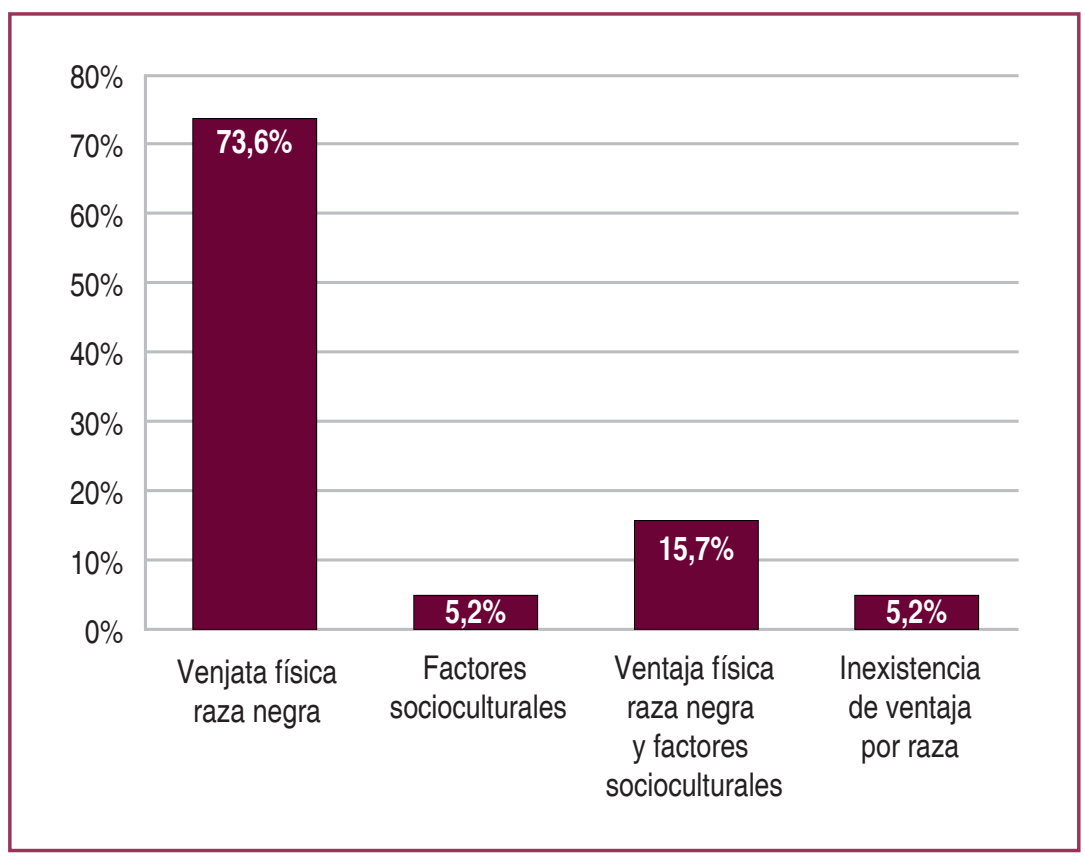

Fuente: Elaboración propia

Para una gran mayoría de los entrevistados el éxito deportivo de los atletas negros se debe, exclusivamente, a factores biológicos, anatómicos y fisiológicos, ya que están genéticamente mejor dotados para las carreras de velocidad.

b. Baloncesto. Respecto al baloncesto $(n=39)$, y en relación con la subcategoría "ventaja física raza negra", el éxito deportivo de los atletas negros se debe, sobre todo, a factores genéticos, anatómicos y fisiológicos. A su vez, un gran número de unidades textuales subrayan la existencia de una superioridad general, en términos biológicos/genéticos: "La predominancia de los deportistas negros en el baloncesto se debe a características genéticas "(estudiante/entrenador 23).

Se recogen, además, unidades textuales $(n=18)$ referidas a elementos como la estructura muscular. Para los entrevistados estos atletas tienen un sistema muscular más adecuado para la práctica de esta disciplina: "Los negros tienen un mayor porcentaje de fibras blancas, por lo cual pueden desenvolverse mejor en gestos explosivos como el salto en el baloncesto" (entrenador 25).

Se han identificado, asimismo, unidades textuales $(n=16)$ referidas a la estructura corporal, señalando la existencia de aspectos propios que podrían ser ventajosos para la práctica deportiva: "La mayor parte de ellos son muy altos" (entrenador 27).

Respecto de la categoría "cultura", subcategoría "factores socioculturales" $(n=7)$, los entrevistados señalan la popularidad de la práctica deportiva como motivo del éxito de los atletas negros en baloncesto: "Las estructuras (campo y canastas) están presentes en cada barrio, así se consigue, también, en las clases menos pudientes (afro-americanas) poder cultivar las propias pasiones" (estudiante 23).

En la subcategoría "factores motivacionales" se descubre un número significativo de unidades textuales $(n=7)$ respecto de la búsqueda de reconocimiento social como, por ejemplo, la motivación que provoca el triunfo deportivo. Este deseo, percibido en algunos colectivos étnicos, es el motivo por el que, según los entrevistados, tienen un mayor éxito estos deportistas 
en baloncesto: "El baloncesto en USA es el deporte más practicado, también en la escuela. Es practicado por mucha gente que tiene ganas de triunfar y por esto aumenta notablemente la posibilidad de emerger" (entrenador 37).

Como demuestran las unidades textuales (figura 2), el 53\% relaciona el éxito de los deportistas negros en baloncesto con "ventaja física raza negra". Desde otro punto de vista, para el $13 \%$, el éxito se debe a factores de tipo natural, pero no asociados a la raza (talento individual independientemente del grupo étnico). Otro 13\% lo justifica por motivos de tipo sociocultural y motivacional. Y, finalmente, el $21 \%$ restante indica que el triunfo se debe, tanto a factores naturales como culturales: ventaja fisica raza negra y factores motivacionales $(13 \%)$; ventaja fisica raza negra y factores socioculturales (8\%).

Figura 2 - Motivos de éxito deportivo: baloncesto

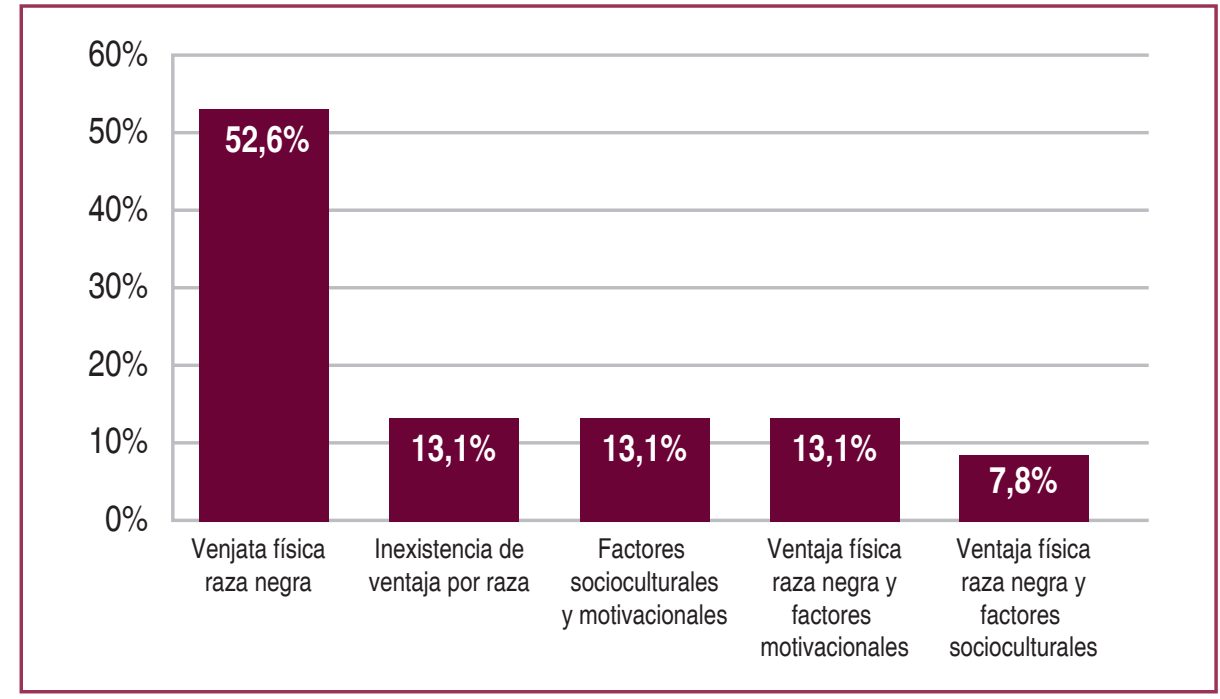

Fuente: Elaboración propia

c. Natación. En relación a la subcategoría "desventaja física raza negra" ( $n=26)$, los participantes han destacado una menor predisposición genética de estos deportistas para la natación: "De sus características genéticas depende la predisposición para los deportes de velocidad"(baloncesto, 100 metros...), "en una indisposición para otros (la natación por ejemplo)" (entrenador 48).

Algunos participantes $(n=9)$ señalan que la dificultad para la natación de los deportistas negros se debe a su mayor densidad ósea: "Los negros tienen una mayor densidad ósea respecto a los blancos (debido a dimensiones mayores de los huesos)" (entrenador 44).

Otras afirmaciones $(n=4)$ están referidas al escaso porcentaje de tejido graso: "Los negros en la natación tienen desventaja por razones físicas. Respecto a otras poblaciones tienen menor porcentaje de tejido graso: por esto flotan menos" (entrenador 46).

Consideran poco adecuado el sistema muscular de los negros para la natación $(n=10)$, los deportistas negros poseen un porcentaje de fibras rojas menos que los atletas blancos: "Tienen una mayor proporción de fibras musculares rápidas, esta predisposición para los esfuerzos rápidos e intensos, ideal para correr los 100 metros en 10"o machacar el balón en la canasta. En la natación, sin embargo, la competición más breve, los 50 metros requieren más de 20" (entrenador 35). 
En el análisis de la categoría "cultura", dentro de la subcategoría "factores socioculturales" $(n=15)$, existen unidades relacionadas con la existencia de una falta de cultura, en las comunidades de origen africano, en relación con la natación, que no favorece la iniciación de los jóvenes en esta práctica dificultando el éxito y su participación: "Los chicos de color prefieren jugar al baloncesto, a fútbol-americano... Es difícil encontrar un joven negro que practiquen natación. No estoy de acuerdo con que tienen músculos diferentes; no vencen, porque no tienen interés en participar" (entrenador 45).

Refiriéndose a la subcategoría "factores socioeconómicos" $(n=6)$, se considera que una de las razones importantes para no practicar la natación es su estatus económico inferior, lo que determina su escaso acceso equipamientos y buenos entrenadores: "Contribuye al poco éxito en esta disciplina la dificultad por parte de los negros de acceder a las instalaciones donde poder practicar la natación" (estudiante 23).

Para el $42 \%$ de los entrenadores (figura 3) no existe una predisposición natural de las personas negras hacia la natación por desventaja física propias de su raza. A su vez, el 34,2 $\%$ afirma que existen, de manera exclusiva, motivaciones de tipo cultural/ambiental, también, propias de esta raza. Finalmente, el $13 \%$ considera que el motivo obedece a factores culturales y naturales.

Figura 3 - Motivos del escaso éxito de los deportistas de raza negra: natación

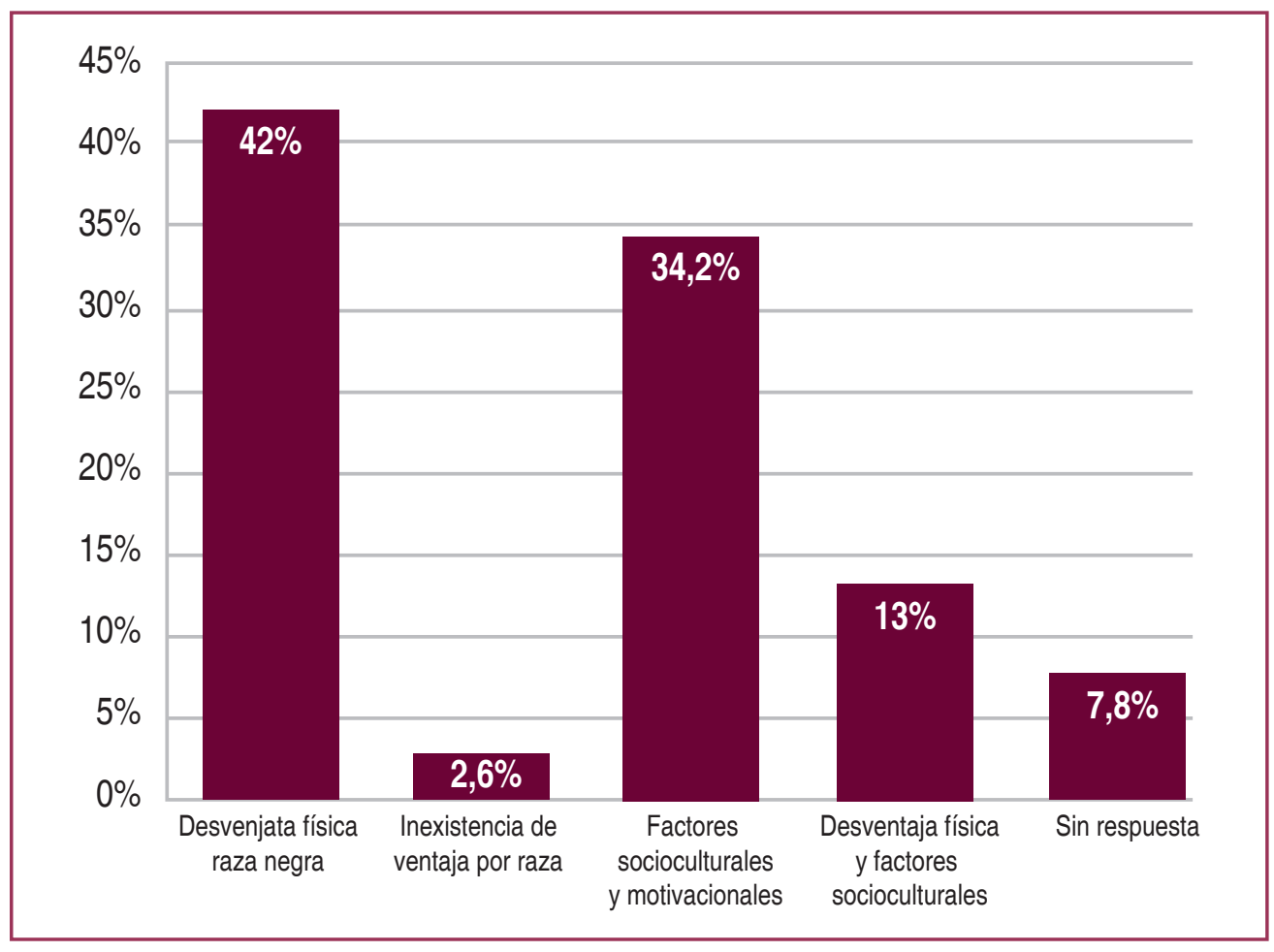

Fuente: Elaboración propia

\section{RESULTADOS DE LA FASE CUANTITATIVA}

a. Carrera de velocidad. La figura 4 recoge los resultados de los cuestionarios con los estudiantes de Ciencias Motoras de las Facultades del Lacio, en relación a la carrera de velocidad y entre los factores que han obtenido un consenso superior al $60 \%$ de los encuestados se encuentran: el biológico y cultural/ambiental. 
Figura 4 - Factores que han recibido mayor consenso: carrera de velocidad

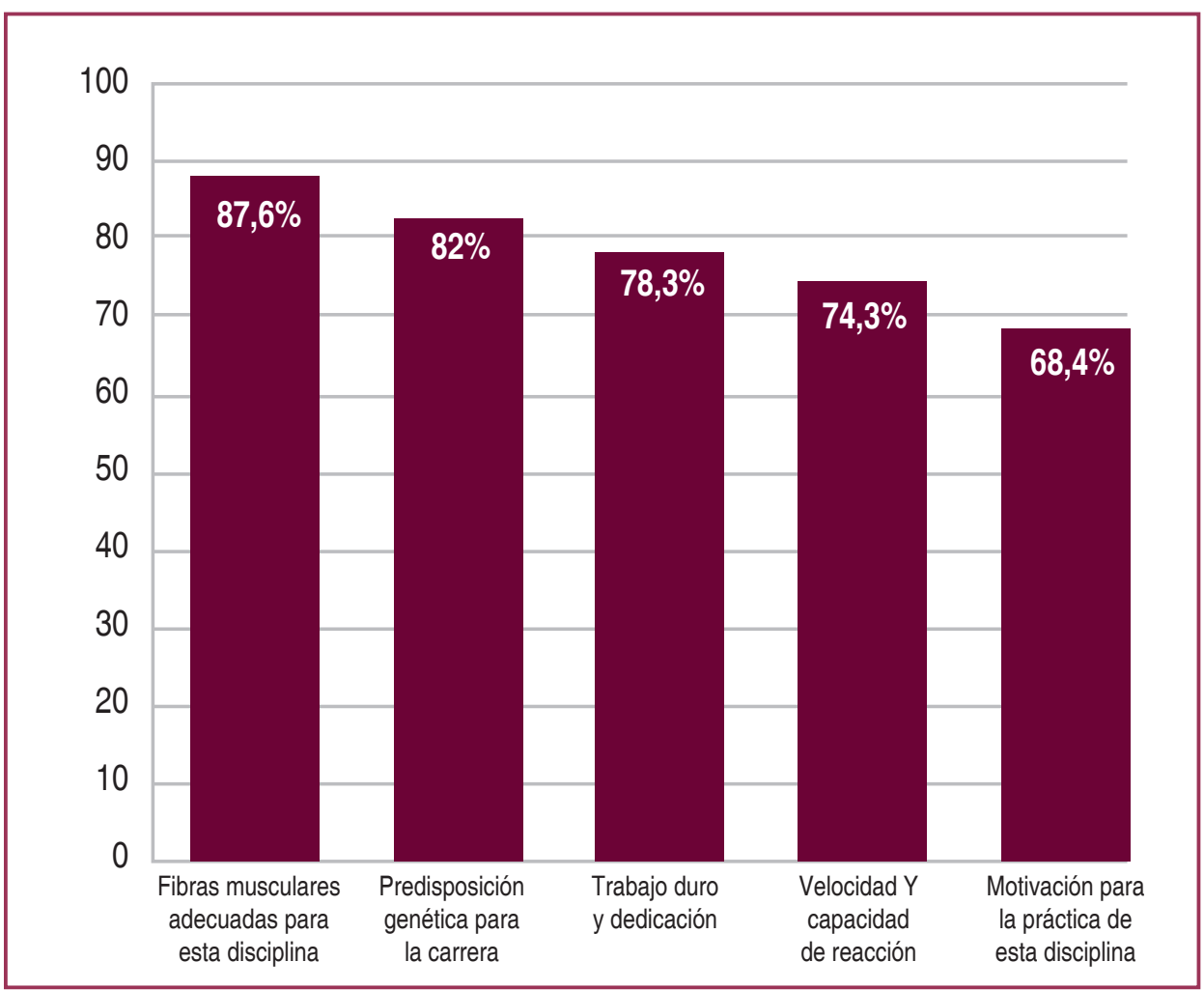

Fuente: Elaboración propia

De esos resultados se deduce que los factores "fibras musculares" (más del $87 \%$ de los participantes) y "predisposición genética para la carrera" (82\%) son registros de carácter biológico/genético altamente valorados. También destaca el factor "trabajo duro y dedicación" (78\%), más propio del ámbito cultural.

b. Baloncesto. En relación con el baloncesto, seis son los factores que han registrado un porcentaje superior al $60 \%$ (figura 5 ).

Figura 5 - Factores que han recibido mayor consenso: baloncesto

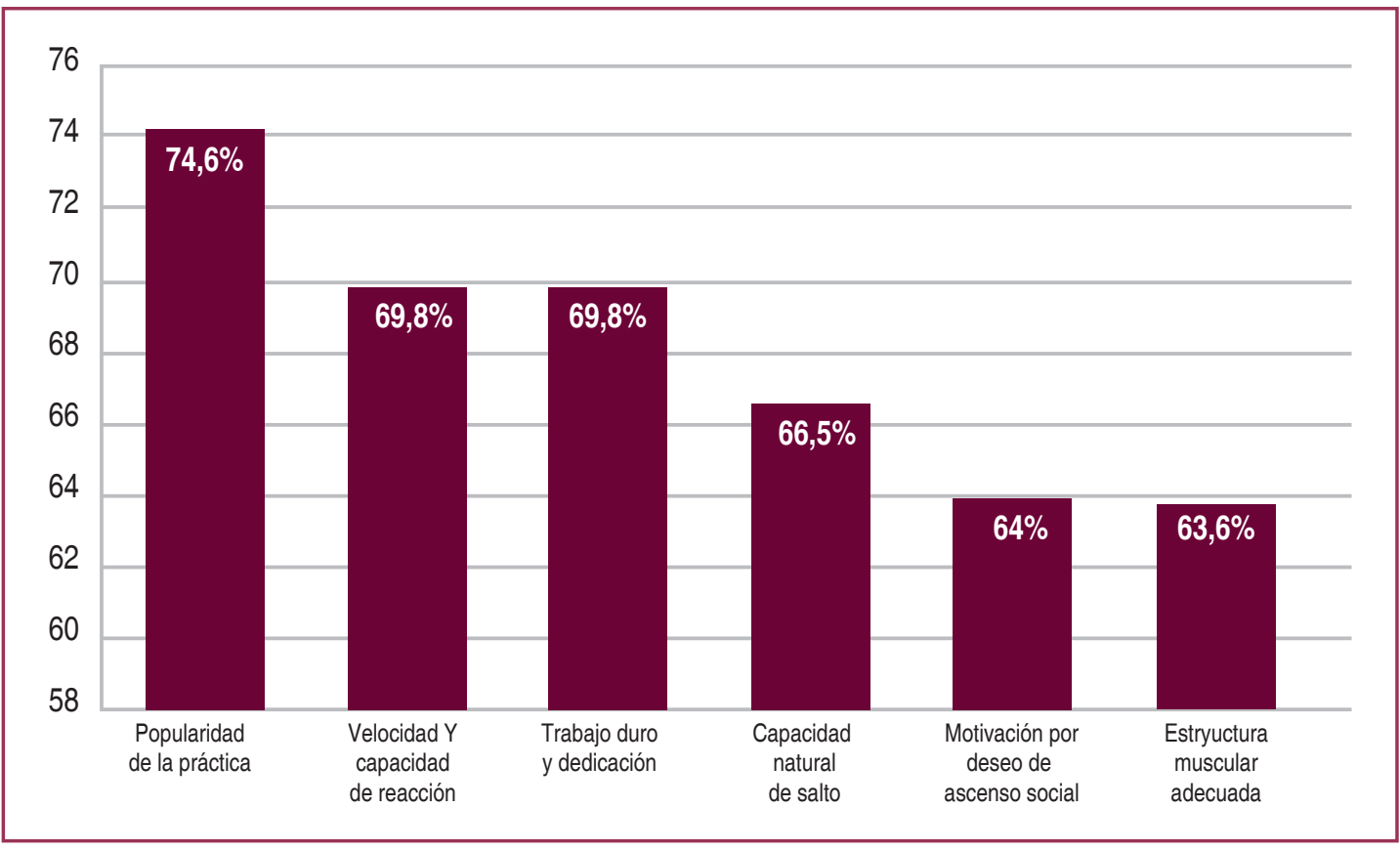

Fuente: Elaboración propia 
Existe cierta paridad entre los factores de carácter "natural": velocidad y capacidad de reacción $(69,8 \%)$, capacidad natural de salto $(66,5 \%)$ y estructura muscular adecuada $(63,6 \%)$, y los de carácter "cultural": popularidad de la práctica $(74,6 \%)$, trabajo duro y dedicación $(69,8 \%)$ y motivación en la práctica por ascenso social (64\%), lo que constituye una ventaja a favor de los deportistas negros. También apreciamos un alto consenso en los factores genéticos (figura 6).

Figura 6 - Consenso registrado por los factores de tipo genético

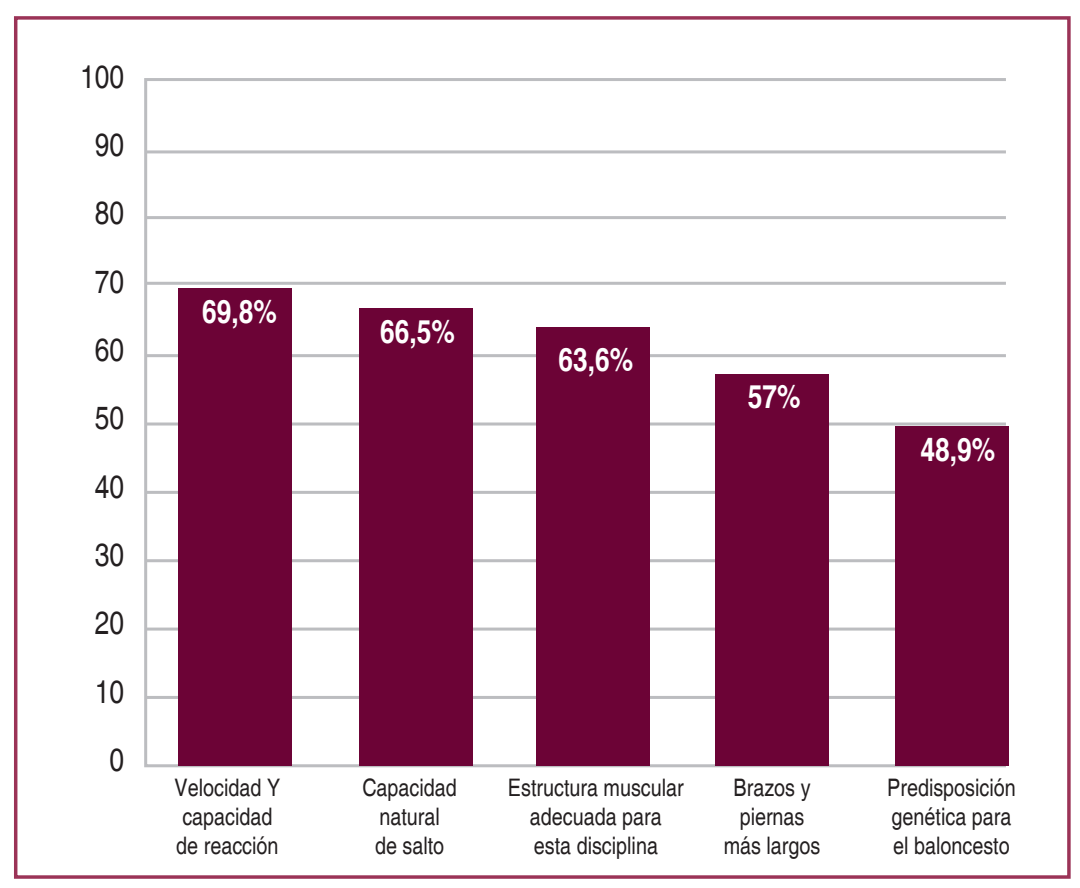

Fuente: Elaboración propia

c. Natación. Según la figura 7 ningún factor obtiene un valor superior al $60 \%$. No obstante, destacan la falta de una cultura de la natación en las comunidades de color, la escasa posibilidad de acceder a mejores instalaciones/entrenadores y, por último, las dificultades de acceso para dicha práctica.

Figura 7 - Factores que han recibido mayor consenso: natación

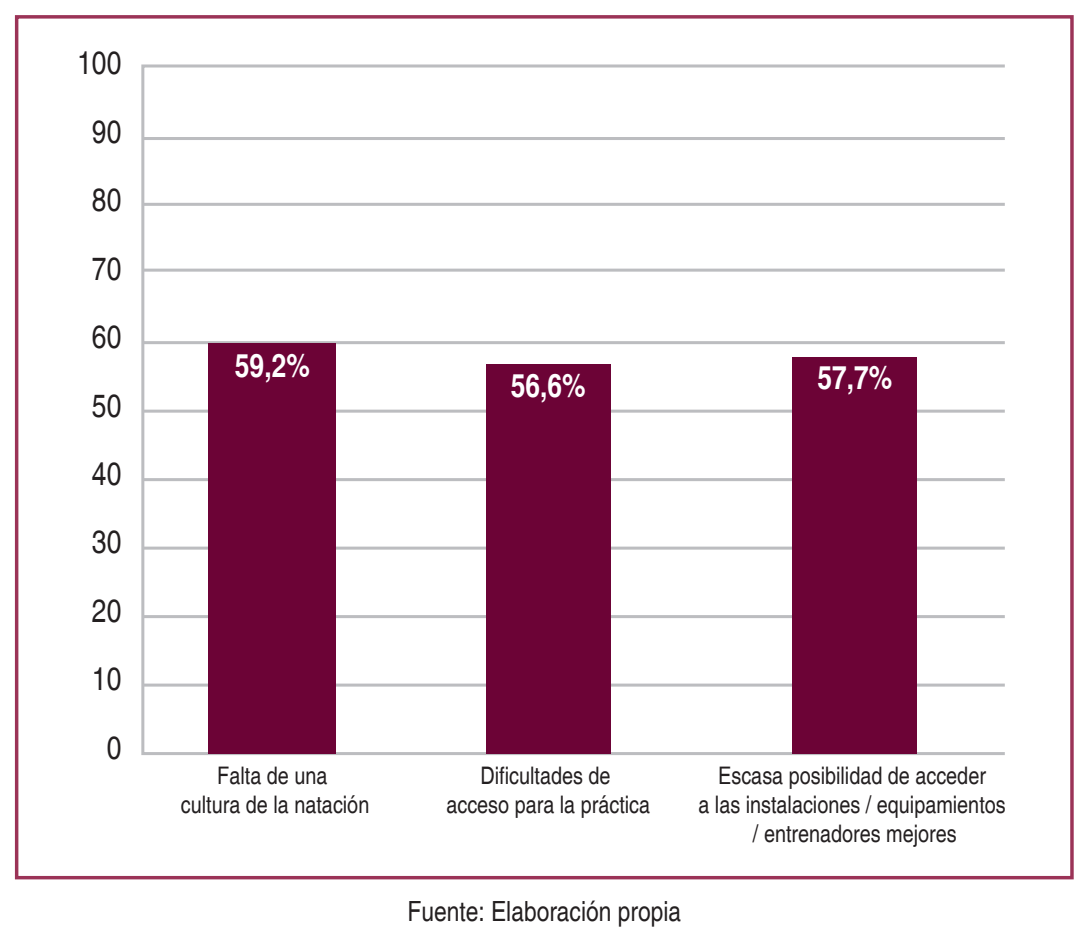


Para más de un tercio de la muestra, los deportistas negros presentan una escasa predisposición genética para la natación $(34,9 \%)$, una estructura muscular $(27,2 \%)$ y corporal $(26,9 \%)$ no adecuadas para esta disciplina, una densidad ósea más elevada y, en consecuencia, escasa habilidad de flotabilidad $(25,0 \%)$ y un menor porcentaje de tejido graso parejo a poca flotabilidad (19,5\%).

\section{DISCUSIÓN Y CONCLUSIONES}

Los resultados obtenidos muestran que los entrenadores y estudiantes de Ciencias Motoras de Lacio poseen estereotipos étnicos ligados a la influencia que su pertenencia étnica ejerce sobre el rendimiento deportivo. Sobre la superioridad atlética de los deportistas negros, la mayor parte de los entrenadores señalan que su éxito se debe, fundamentalmente, a una ventaja física por "raza"; es decir, por pertenecer a un grupo étnico percibido como superior de manera natural (factores de tipo biológico-genético). Esta creencia en la superioridad innata de las personas negras coincide con los estudios de Harrison; Lawrence (2004); Sheldon, Jayaratne, Petty (2007); Azzarito; Harrison (2008); Harrison et al. (2011).

El reconocimiento de la contribución genética es un factor que se destaca en el estudio cualitativo de Harrison e Lawrence (2004), aunque, en nuestro caso, los datos revelan la presencia de un estereotipo más extendido. El 25\% de los participantes ha declarado el éxito de los deportistas negros en términos de ventaja física ligada a aspectos genéticos, estructurales y fisiológicos. En este sentido, el 73,6\% de los entrevistados (sobre la carrera) y el 52,6\% (sobre el baloncesto) responden que el éxito de los deportistas negros se debe a una ventaja física exclusivamente de carácter genético-biológico. Este porcentaje se ve incrementado (89,3\%) en la carrera y $(73,5 \%)$ en el baloncesto cuando se consideran las respuestas mixtas, en las que se reconoce que el éxito en estas disciplinas se debe tanto a razones genéticas como culturales.

De igual manera Azzarito e Harrison (2008), indican cómo los estudiantes americanos de Educación Física creen en la superioridad atlética natural de los negros, considerándolos, biológicamente, diferentes a los blancos y físicamente superiores. Sin embargo, para Harrison et al. (2011) esta diferencia genética entre los deportistas blancos y negros es considerada sólo por el $17 \%$ de los estudiantes universitarios.

Este estereotipo de superioridad natural de los deportistas negros, no sólo se descubre en el ámbito americano sino, también, en el contexto europeo. Nuestros resultados coinciden con los profesores de Educación Física ingleses (HAYES; SUDGEN, 1999). Así, para el 82\% los alumnos negros presentan ventajas en algunos deportes (atletismo, baloncesto, fútbol, cricket y boxeo) y el $74 \%$ cree que el éxito de los negros se debe a razones fisiológicas. Resultados que coinciden con nuestro estudio cualitativo (el $73,6 \%$ lo relaciona con la carrera y el $52,6 \%$ ligado al baloncesto) y cuantitativo. En concreto, la mayoría de los estudiantes universitarios consideran que la superioridad física (aspectos fisiológicos de los deportistas negros) es un factor fundamental para su éxito deportivo en el atletismo (70\%) y en el baloncesto (65\%). De este estudio inglés se concluye que entre los factores citados de tipo genético, los de mayor porcentaje se vinculan con el papel destacado de las fibras musculares y su influencia a favor de una mejora de la velocidad y de una mayor masa muscular, elementos relevantes para el éxito deportivo de los negros, visibles y coincidentes en ambas fases de nuestro estudio. 
La creencia de una mayor predisposición genética en algunas disciplinas deportivas tiene una gran presencia en nuestra investigación. Se confirma la creencia en la superioridad y predisposición natural de los deportistas negros para el baloncesto, en sintonía con los estudios de Johnson, Hallinan, Westerfield (1999); Harrison e Lawrence (2004), y para la carrera de velocidad, todo ello de acuerdo con Rasmussen, Turner, Esgate (2005) y Turner e Jones (2007).

Los deportistas negros desarrollan una velocidad mucho más natural y poseen una estructura muscular más adecuada para la carrera corta. La mayoría de los participantes estima que la habilidad atlética natural de los deportistas negros se debe a la composición y estructura muscular; concretamente, al tipo de fibras musculares (87,6\%). En este sentido, Sailes (2009) confirma la existencia de la idea estereotipada de que los deportistas negros tienen un mayor número de fibras musculares blancas (veloces), mientras que en los blancos predominan las fibras rojas (lentas). Los resultados obtenidos demuestran que aún sigue vigente esta idea estereotipada nacida de investigaciones anteriores.

El análisis de los datos en baloncesto muestra cómo la mayoría atribuye el triunfo de los deportistas negros a factores exclusivamente biológicos. No obstante, los que atribuyen el éxito deportivo a factores no biológicos (socioculturales y motivacionales) es mayor en relación con la carrera de velocidad.

Los factores culturales, la práctica del entrenamiento y el deseo por ascender socialmente son percibidos como aspectos fundamentales en el éxito de los deportistas negros (HARRISON; LAWRENCE; BUKSTEIN, 2011; TURNER; JONES, 2007; AZZARITO; HARRISON, 2008; HARRISON: LAWRENCE, 2004), sin olvidarnos de algunos elementos biológico-genéticos. Por ello, un $70 \%$ de los estudiantes considera que los deportistas negros muestran una mayor velocidad y un $67 \%$ una mayor capacidad natural de salto. Igualmente, más del 50\% valoran la estructura muscular de los deportistas negros (a nivel de brazos y de piernas), como más adecuados para el baloncesto. Así, pues, el estereotipo más difundido se relaciona con aspectos genéticos (cantidad de fibras musculares) y con una mayor potencia y velocidad.

Sobre la natación, es un número similar los que señalan la existencia de ciertas desventajas de los nadadores negros por motivos naturales y aquellos que las atribuyen a causas de tipo cultural (sociocultural o socioeconómico), aspectos que no se reflejan en otras disciplinas. Su morfología habitual (masa muscular, tejido graso y densidad ósea) es, según los entrevistados, la causa de una menor flotación, provocando menor éxito en la natación. Esto certifica la creencia entre algunos entrenadores y estudiantes sobre su escasa predisposición para la natación.

Algo menos de la mitad (42\%), atribuyen el escaso éxito de los nadadores negros a factores exclusivamente biológicos, sin considerar elementos culturales y ambientales. Esta creencia errónea está difundida en el mundo de la natación debiendo analizar el origen, las causas y sus posibles influencias en los participantes (jóvenes negros). Ese posible estereotipo cultural-socioeconómico, puede determinar que los estudiantes relacionen al deportista negro con un nivel socioeconómico inferior y con limitaciones para acceder a las instalaciones deportivas, lo que puede dificultar su éxito en esta disciplina. Esta percepción, según Burden, Hodge, Harrison Junior (2004), indica que los estudiantes americanos tienen creencias estereotipadas sobre la natación, al considerar a los nadadores negros menos hábiles que los blancos. 
En este estudio se destaca la presencia de estereotipos étnicos, en el ámbito deportivo, que justifican la necesidad de analizar las posibles implicaciones pedagógicas (MIGLIORATI, 2011). El estereotipo del negro, como atleta "excelente", puede ser tan predominante y tan poderoso hasta el punto de marcar la autodefinición que los negros dan de su propia identidad. Esto podría justificar la hipótesis de algunos investigadores, al señalar que las comunidades afroamericanas utilizan el deporte para mejorar su estatus social.

Los estereotipos étnicos, ciertamente, pueden influir en la calidad de las prestaciones. Así, los deportistas blancos asumirán las consecuencias del estereotipo negativo que los considera menos capacitados para saltar y, en consecuencia, se dedicarán en menor medida 0 con menores expectativas al baloncesto. En el lado opuesto, el convencimiento difundido entre muchos deportistas negros sobre sus capacidades físicas y atléticas podría ser la clave para consolidar su superioridad.

Los educadores físicos con visiones estereotipadas sobre la pertenencia étnica de los deportistas pueden tener expectativas diferentes sobre las habilidades y las capacidades de estos, lo que puede determinar una mayor o menor confianza del entrenador hacia sus deportistas generándose diferentes expectativas. Estos educadores, a partir de sus convicciones (a menudo falsas, como muchos estereotipos), pueden reforzar el mito de que existe una diversidad genética importante entre los diferentes grupos étnicos. Además, pueden influir en el aprendizaje de los deportistas hasta el punto de marcar su autopercepción y, en consecuencia, su performance, sus expectativas hacia el deporte e incluso su desarrollo personal.

Por tanto, elámbito deportivo podría originar discriminación, intolerancia e incomprensión entre diferentes grupos étnicos. Los estereotipos pueden subestimar las diferencias individuales y enfatizar las diferencias entre los distintos grupos étnicos, siendo necesario propuestas educativas para una mejor convivencia social y de atención a la diversidad. Siendo necesario eliminar la presencia de estereotipos étnicos en el entorno educativo y deportivo. Para ello, al entrenador se le debe reconocer su rol educativo en la formación de los deportistas y en la transmisión de valores.

Por último, las funciones pedagógicas de los educadores deportivos deberán ser reformuladas y reelaboradas desde una perspectiva intercultural, siendo preciso convertir el deporte en un medio de inclusión social, liberado de cualquier estereotipo y prejuicio étnico.

\section{REFERENCIAS}

ALLPORT, Gordon W. The nature of prejudice. Cambridge: Addison-Wesley, 1954.

AZZARITO, Laura; HARRISON JUNIOR, Louis. "White men can't jump". Race, gender, and natural athleticism. International Review for the Sociology of Sport, London, v. 43, n. 4, p. 347-364, 2008.

BARBUJANI, Guido. L'invenzione delle razze. Milano: Bompiani, 2006.

BURDEN JÚNIOR, Joe W.; HODGE, Samuel R.; HARRISON JUNIOR, Louis. African american and white american students' beliefs about ethnic groups' aspirations: a paradoxical dilemma of academic versus athletic pursuits. E-Journal of Teaching and Learning in Diverse Settings, Baton Rouge, v. 2, n. 1, p. 54-77, 2004.

HARRISON, C. Keith; LAWRENCE, Suzanne Malia. College students' perceptions, myths, and stereotypes about African American athleticism: a qualitative investigation. Sport, Education and Society, London, v. 9, n. 1, p. 33-52, 2004. 
HARRISON, C. Keith; LAWRENCE, Suzanne Malia; BUKSTEIN, Scott J. White College Students' Explanations of White (and Black) Athletic Performance: A Qualitative Investigation of White College Students. Sociology of Sport Journal , Champaign, v. 28, n. 3, p. 347-361, 2011.

HARRISON JUNIOR, Louis. Understanding the influence of stereotypes: Implications for the African American in sport and physical activity. Quest, New York, v. 53, n. 1, p. 97-114, 2001.

HARRISON JUNIOR, Louis; AZZARITO, Laura; BURDEN, Joe W. Jr. Perceptions of athletic superiority: A view from the other side. Race, Ethnicity and Education, Philadelphia, v. 7, n. 2, p. 149-166, 2004.

HAYES, Sid; SUGDEN, John. Winning through "naturally" still? An analysis of the perceptions held by physical education teachers toward the performance of black pupils in school sport and in the classroom. Race, Ethnicity and Education, Philadelphia, v. 2, n. 1, p. 93-108, 1999.

HODGE, Samuel R. et al. A comparison of high school students' stereotypic beliefs about intelligence and athleticism. Educational Foundations, San Francisco, v. 22, n. 1-2, p. 99-119, 2008.

HOBERMAN, John. Darwin's Athletes: how sport has damaged black America and preserved the myth of race. New York: Houghton Mifflin, 1997.

HORN, Thelma Sternberg; LOX, Curt L. The self-fulfilling prophecy theory: When coaches' expectations become reality. In: WILLIAMS, Jean Marie (Ed.). Applied Sport Psychology. Mountain View: Mayfield, 1993. p. 68-81.

HUNTER, David W. Race and athletic performance: A physiological review. In: SAILES, Gary A. (Ed.), African Americans in Sport: Contemporary Themes. New Brunswick, NJ: Transaction, 2009. p. 85-101.

JOHNSON, Darrel L.; HALLINAN, Christhoper J.; WESTERFIELD, R. Carl. Picturing Success:

Photographs and Stereotyping in Men's Collegiate Basketball. Journal of Sport Behavior, Mobile, v. 22, n. 1, p. 45-53, 1999.

LLEIXÁ, Teresa. Actividad física, deporte y ciudadanía intercultural. In: LLEIXÁ, Teresa; PRAT, Susanna. (Ed.). Actividad física y deporte en sociedades multiculturales. ¿Integración o segregación? Barcelona: Horsori, 2004. p. 13-26.

MACIONIS, John Joseph. Sociología. Madrid: Pearson Prentice Hall, 2007.

MAZZARA, Bruno M. Stereotipi e pregiudizi. Bologna: II Mulino, 1997.

MIGLIORATI, Mascia. Los estereotipos en el deporte y sus implicaciones pedagógica. In: ISIDORI, Emanuele; FRAILE, Antonio (Ed.). La pedagogía del deporte hoy: escenarios y desafíos. Roma: Nuova Cultura, 2011. p. 135-170.

MIGLIORATI, Mascia. Allenamento e interculturalità. Prospettive pedagogiche per la formazione degli educatori sportivi. In: ISIDORI, Emanuele; FRAILE, Antonio (Ed.). Pedagogia dell'allenamento: prospettive metodologiche. Roma: Nuova Cultura, 2012. p. 207-226.

RASMUSSEN, Ricky; TURNER, David; ESGATE, Anthony. On your marks, get stereotyped, go. Novice coaches and black stereotypes in sprinting. Journal of Sport and Social Issues, London, v. 29, n. 4, p. 426-436, 2005.

REFRIGERI, Luca. Lo sport agenzia non formale per l'educazione antirazzista. In:

REFRIGERI, Luca (Ed.) Sport e razzismo: il ruolo dell'educazione. Lecce: Pensa Multimedia, 2011. p. 103-128.

SAILES, Gary A. An investigation of campus stereotypes: the myth of black athletic superiority and the dumb jock stereotype. Sociology of Sport Journal, Champaign, v. 10, n. 1, p. 88-97, 1993.

SAILES, Gary A. The african american athlete: Social myths and stereotypes. In: SAILES Gary A. (Ed.)

African Americans in sport Contemporary Themes. New Brunswick, NJ: Transaction, 2009. p. 183-198. 
SHELDON, Jane P.; JAYARATNE, Toby Epstein; PETTY, Elizabeth M. White Americans' genetic explanations for a perceived race difference in athleticism: The relation to prejudice toward and stereotyping of Blacks. Athletic Insight, v. 9, n. 3, p. 31-56, 2007.

STONE, Jeff., LYNCH, Christian. I., SJOMELING, Mike., \& DARLEY, John. M. (1999). Stereotype Threat Effects on Black and White Athletic Performance. Journal of Personality and Social Psychology, New York, v. 77, n. 6. p. 1213-1227.

TURNER, David; JONES, lan. False start? U.K. Sprint coaches and black/white stereotypes. Journal of Black Studies, London, v. 38, n. 2, p. 155-176, 2007.

WIGGINS, David K. "Great speed but little stamina": The historical debate over black athletic superiority. Journal of Sports History, Champaign, v. 16, n. 2, p. 158-185, 1989. 